\title{
Age Differences in Social Comparison Tendency and Personal Relative Deprivation
}

\author{
Mitchell J. Callan \\ Department of Psychology, University of Essex, Colchester, United Kingdom \\ Hyunji Kim \\ Department of Psychology, University of Essex, Colchester, United Kingdom \\ William J. Matthews \\ Department of Psychology, University of Cambridge, Cambridge, United Kingdom
}

\section{Word count: 2,985}

Author note: This research was funded by a grant from the Leverhulme Trust (RPG-2013148). Address correspondence to Mitchell J. Callan, Department of Psychology, University of Essex, Wivenhoe Park, Colchester, United Kingdom, CO4 3SQ (mcallan@essex.ac.uk). 


\begin{abstract}
We examined age-related differences in social comparison orientation and personal relative deprivation (PRD). In Study 1, participants $(N=1,290)$ reported their tendencies to engage in social comparisons and PRD. Older adults reported lower levels of social comparison tendency and PRD, and social comparison tendency mediated the relation between age and PRD. The findings reported in Study 1 were replicated in Study 2 using a sample of participants between the ages of 18 to $30(n=180)$ and $60+$ years old $(n=176)$. Our findings provide evidence that older adults report lower levels of social comparison tendency that, in turn, relate to lower levels of PRD.
\end{abstract}

Keywords: aging; social comparison; social comparison orientation; personal relative deprivation 


\section{Age Differences in Social Comparison Tendency and Personal Relative Deprivation}

\section{INTRODUCTION}

Often the only way we can determine the adequacy of our opinions and abilities is through social comparison, and we typically compare ourselves with people who are similar to us, because similar others provide the most diagnostic information for self-evaluation (Festinger, 1954). Although learning about the self through social comparison is ubiquitous (Corcoran, Crusius, \& Mussweiler, 2011), Suls and Mullen (1982) proposed that the tendency to engage in social comparison weakens across adulthood, peaking around young adulthood and decreasing from middle age to older age. They argued that older adults tend to engage in social comparisons less often than younger adults due to losses of, or shifts in, interpersonal contacts, social isolation, general disengagement from society, and declines in the cognitive capacities required for social comparison (e.g., perspective taking).

Little empirical attention has been given to the development of social comparison throughout adulthood, and the evidence Suls and Martin (1982) used to support their model was indirect (e.g., evidence pointing to the effects of age on social isolation). Researchers have examined the role of social comparison processes in psychological adjustment among older adults (e.g., Heidrich \& Ryff, 1993; Robinson-Whelen \& Kiecolt-Glaser, 1997), but direct evidence pointing to age-related differences in the tendency to socially compare is limited. Our first aim, then, was to explore the relation between age and tendencies to engage in social comparisons.

Our second aim was to explore one potential consequence of a weaker tendency to engage in social comparisons among older adults — namely, reduced personal relative deprivation (PRD). PRD refers to resentment originating from the belief that one is deprived of desired and deserved outcomes compared to others (for a review, see Smith, Pettigrew, Pippin, \& Bialosiewicz, 2012). As Smith et al. (2012) outlined, PRD is characterized by a 
process whereby an individual makes a social comparison on a given outcome, believes themself to be comparatively disadvantaged, and consequently feels resentful. Despite being an important predictor of a range of outcomes (Smith et al., 2012), to our knowledge, little research has examined age-related differences in PRD. Given that experiences of PRD by definition require social comparison, social comparison tendency should positively correlate with PRD (cf. Buunk, Zurriaga, Gonzalez-Roma, \& Subirats, 2003). Consequently, if older adults report weaker tendencies to engage in social comparisons, as Suls and Martin (1982) suggest, then they might also feel less relatively deprived.

\subsection{Overview of Research}

Across two studies we examined the relations among age, tendencies to engage in social comparisons, and PRD. For Study 1, we collated data across four existing studies where we measured participants' age, tendencies to engage in social comparisons, and PRD. Study 2 was a confirmation study where we recruited participants between the ages of 18 and 30 and over 59 years old to investigate age differences in social comparison tendencies of abilities and opinions and PRD.

\section{STUDY 1}

\subsection{Method}

\subsubsection{Participants}

Participants were 1,290 adults from the USA $\left(M_{\text {age }}=36.55\right.$, Age range $=18$ to $83 ; S D_{\text {age }}=$ 12.24; $57 \%$ female) who completed a brief online survey through Amazon's Mechanical Turk (MTurk) or CrowdFlower.com for a nominal payment.

\subsubsection{Procedure and measures}

We collated data across four unpublished studies that explored the antecedents and consequences of PRD. Along with various other measures depending on the aims of the individual studies, participants across samples completed Gibbons and Buunk's (1999) 
widely-used 11-item Iowa-Netherlands Comparison Orientation Measure (INCOM). The items concern tendencies to engage in ability- and opinion-based social comparisons (e.g., "I always pay a lot of attention to how I do things compared with how others do things"; "I often like to talk with others about mutual opinions and experiences"). Participants rated the items using a 5-point scale $(1=$ disagree strongly to $5=$ agree strongly). Responses were averaged across items (two items were reverse-scored) per Gibbons and Buunk's (1999) recommended use of the INCOM; higher scores indicate stronger tendencies to engage in social comparisons.

Participants also completed Callan, Shead, and Olson's (2011) 5-item Personal Relative Deprivation Scale (PRDS), which gauges individual differences in people's beliefs and feelings associated with comparing their outcomes with the outcomes of similar others (e.g., "I feel dissatisfied with what I have compared to what other people like me have"). The PRDS has been shown to predict theoretically relevant consequences of PRD (e.g., selfesteem, delay discounting, see Callan, Ellard, Shead, \& Hodgins, 2008; Callan et al., 2011). Participants responded to the items using a 6-point scale $(1=$ strongly disagree, $6=$ strongly agree). Responses were averaged across items (two items were reverse-scored); higher scores indicate more PRD.

\subsection{Results}

\subsubsection{Preliminary data analyses}

A series of moderated regression analyses showed that the associations among age, PRD, and tendency to socially compare did not differ significantly by sample (i.e., there were no significant interactions; all $p \mathrm{~s}>.26$ ). Accordingly, we collated the data across the four samples for our main analyses.

\subsubsection{Correlation and mediation analyses}


Table 1 shows descriptive statistics, alpha reliabilities, and correlations among the measures.

Older participants reported weaker tendencies to socially compare and lower PRD. As expected, a greater general tendency to socially compare related to higher PRD.

Using Preacher and Hayes's (2008) bootstrapping procedure for testing indirect effects, we tested the indirect effect of age on PRD through tendency to socially compare (see Figure 1). This analysis revealed that social comparison orientation mediated the relation between age and PRD (10,000 resamples; indirect effect $=-.004,95 \%$ bias-corrected and accelerated confidence interval $[\mathrm{BCa} \mathrm{CI}]:-.006,-.003)$, suggesting that that one reason why older adults experience less PRD is through a weaker tendency to engage in social comparisons.

\section{STUDY 2}

Given the exploratory nature of Study 1, we conducted a confirmation study where we administered the INCOM and PRDS to younger (18-30) and older (60+) adults. Although Gibbons and Buunk (1999) advocated the use of the full 11-item INCOM to gauge general social comparison tendencies, Schneider and Schupp (2014) recently found that a two factor model — with the ability and opinion subscales as distinct but correlated factors—was superior to the one factor model. In another study we conducted prior to the current Study 2, we recruited younger (18-30) and older (60+) adults and administered the PRDS and 11-item INCOM. Along with finding age differences in PRD and tendencies to engage in social comparisons of abilities and opinions, we corroborated Schneider and Schupp's (2014) findings for the two-factor model. Full details of this study are available in the supplementary content.

Accordingly, using a 6-item version of the INCOM (hereafter INCOM-6) validated by Schneider and Schupp (2014), Study 2 examined age differences in social comparisons of abilities and opinions and PRD. We expected that the older adults would report a weaker 
tendency to socially compare in terms of abilities and opinions, and lower PRD, and that tendency to socially compare would mediate age-related differences in PRD. Because social comparisons of abilities (which concern self-evaluative questions of "how am I doing?"; Gibbons \& Buunk, 1999) are more relevant to people's perceptions of their relative lot in life than are social comparisons of opinions (which concern questions of "what should I think?"), we expected the relation between age and PRD to operate primarily through social comparisons of abilities (see Table 1: Study 1, and the online supplementary content for exploratory analyses suggesting this pattern).

\subsection{Method}

\subsubsection{Participants}

Participants were 180 younger (18-30) and 176 older (60+) adults living the USA recruited through MTurk. Fifty-seven additional participants were not included because they either completed the survey twice $(n=14)$ or failed an attention check item ("Attention check. Please select "strongly disagree"”; $n=43$ ). The proportion of participants who failed the attention check did not differ between ages (12\% and $8.4 \%$ for older and younger participants, respectively, $p=.18$ ).

\subsubsection{Procedure and measures}

Participants first reported their age to determine their eligibility to participate. Eligible participants then completed the INCOM-6 followed by the PRDS. Participants then reported their annual household income using an 8-point ordinal scale with values ranging from 1 (less than $\$ 15,000$ ) to 8 (greater than \$150,000), coded using Parker and Fenwick’s (1983) median-based estimator. Participants also indicated their highest level of education $(1=$ did not finish high school, 2 = high school graduation, $3=$ college graduation, $4=$ postgraduate degree). Finally, participants provided their gender and ethnicity (White/Caucasian, African American, Hispanic, Asian, Native American, Pacific Islander or Other). Along with gender 
and ethnicity, annual income and educational attainment were included as control variables to test whether age-related differences might be due to differences in socioeconomic status than PRD. See Table 2 for sample characteristics by age.

\subsection{Results}

\subsubsection{Measurement invariance}

Using a model comparison approach (Hair, Black, Babin, \& Anderson, 2010), we first conducted Multi-group Confirmatory Factor Analyses (MCFA) using the lavaan package (v. 0.5-18; Rosseel, 2012) in R to test measurement invariance across age groups for the PRDS and INCOM-6. The PRDS was age invariant in terms of the factor loadings (configural vs. metric model: $\left.\Delta \chi^{2}=3.31, \Delta d f=4, p=.51, \Delta C F I=-.001\right)$ and loadings and intercepts (scalar vs. metric model: $\left.\Delta \chi^{2}=4.07, \Delta d f=4, p=.40, \Delta C F I=.000\right)$. The INCOM-6 was age invariant for the factor loadings $\left(\Delta \chi^{2}=3.99, \Delta d f=4, p=.41, \Delta C F I=.000\right)$ and partially invariant for the loadings and intercepts (i.e., while relaxing the equality of intercepts constraint for the "I am not the type of person who compares often with others" item; $\Delta \chi^{2}=$ $0.31, \Delta d f=3, p=.96, \Delta C F I=-.004)$. Demonstrating at least partial scalar invariance permits meaningful comparisons of latent means between groups (Steenkamp \& Baumgartner, 1998). Full details of these model comparisons are available in the supplementary content.

\subsubsection{Age differences}

Shown in Table 2, the older (vs. younger) adults demonstrated a weaker tendency to engage in social comparisons of abilities, $t(354)=7.63, p<.001, d=.81^{1}$, and social comparisons of opinions, $t(354)=4.70, p<.001, d=.50$. The older participants also reported lower PRD, $t(354)=4.24, p<.001, d=.45$. These age differences remained significant in analyses controlling for income, education, ethnicity (Caucasian vs. other), and gender (all $p$ s $<.001)$. Comparisons of the latent means from the above MCFAs revealed the same pattern

\footnotetext{
${ }^{1}$ There was also a significant difference when using only a composite of the two items from the ability subscale that met the criteria for full scalar invariance, $p<.001, d=.63$.
} 
of age-related differences for PRD $(p<.001, d=.49)$, social comparison of abilities (while allowing the non-invariant intercept to be different, $p<.001, d=.72$ ), and social comparisons of opinions $(p<.001, d=.55)$.

\subsubsection{Mediation analyses}

Bootstrapped multiple mediation analyses showed that social comparison of ability (indirect effect $=-.101,95 \%$ BCa CI: $-.165,-.044$ ), but not social comparison of opinions (indirect effect $=.024,95 \%$ BCa CI: $-.010, .066)$, mediated the effect of age on PRD.

\section{GENERAL DISCUSSION}

Consistent with Suls and Mullen's (1982) life-span model of comparison processes, we found that older adults reported a weaker tendency to socially compare than younger adults. Older adults also reported feeling less personal relative deprivation than younger adults. Our mediation analyses suggest that the relation between age and PRD is due, in part, to older adults reporting weaker tendencies to engage in social comparisons.

These studies show that social comparison orientation weakens across adulthood, and our research is the first to show that these differences - at least for social comparisons of abilities - relates to weaker PRD. The present studies were cross-sectional, leaving open the possibility that the age differences we observed might be due to cohort effects. Longitudinal studies are therefore needed to investigate intra-individual changes in social comparison tendency and PRD. Nonetheless, our results lend impetus to future research exploring the psychological consequences of age-related differences in social comparison tendency and PRD, such as how reduced PRD through social comparisons may contribute to maintaining subjective well-being in later adulthood.

It will also be important for future research to investigate the antecedents and consequences of age-related differences in social comparison orientation and PRD. One possibility is that old age might start a transition toward self-evaluation based more on one's 
personal past than social comparison (Suls \& Mullen, 1982). Insofar as old age brings limited abilities or opportunities to engage in social comparisons, older adults might nonetheless achieve self-assessment through temporal self-comparisons (e.g., through reminiscing; ReisBergan, Gibbons, Gerrard, \& Ybema, 2000). Such changes toward a greater preference for, or frequency of, temporal self-comparisons in older age might also underpin age differences in feelings of resentment arising from adverse social comparisons of abilities. Further, age still exhibited a significant direct effect on PRD across both studies, suggesting that decreased social comparison is not the only reason why PRD decreases among older adults. For example, older adults might feel more sanguine about their comparative disadvantage because of age-related changes in affect regulation (Kessler \& Staudinger, 2009). 


\section{References}

Buunk, B. P., Zurriaga, R., Gonzalez-Roma, V., \& Subirats, M. (2003). Engaging in upward and downward comparisons as a determinant of relative deprivation at work: A longitudinal study. Journal of Vocational Behavior, 62, 370-388.

Callan, M. J., Shead, N. W., \& Olson, J. M. (2011). Personal relative deprivation, delay discounting, and gambling. Journal of Personality and Social Psychology, 101, 955973.

Corcoran, K., Crusius, J., \& Mussweiler, T. (2011). Social comparison: Motives, standards, and mechanisms. In D. Chadee (Ed.), Theories in social psychology (pp. 119-139). Oxford, UK: Wiley-Blackwell.

Festinger, L. (1954). A theory of social comparison processes. Human Relations, 7, 117-140.

Gibbons, F. X., \& Buunk, B. P. (1999). Individual differences in social comparison: Development of a scale of social comparison orientation. Journal of Personality and Social Psychology, 76, 129-142.

Hair, J. R., J., \& Black, W. C., Babin, B. J., \& Anderson, R.E. (2010). Multivariate data analysis (7th ed.). Upper Saddle River, NJ: Prentice Hall.

Heidrich, S. M., \& Ryff, C. D. (1993). The role of social comparisons processes in the psychological adaptation of elderly adults. Journal of Gerontology: Psychological Sciences, 48, 127-136.

Kessler, E. M., \& Staudinger, U. M. (2009). Affective experience in adulthood and old age: The role of affective arousal and perceived affect regulation. Psychology and Aging, 24, 349-362.

Parker, R. N., \& Fenwick, R. (1983). The Pareto curve and its utility for open-ended income distributions in survey research. Social Forces, 61, 872-885.

Preacher, K. J., \& Hayes, A. F. (2008). Asymptotic and resampling strategies for assessing and comparing indirect effects in multiple mediator models. Behavior Research Methods, 40, 879-891. 
Reis-Bergan, M., Gibbons, F. X., Gerrard, M. \& Ybema, J.F. (2000). The impact of reminiscence on socially active elderly women's reactions to social comparison. Basic and Applied Social Psychology, 22, 225-236.

Robinson-Whelen, S., \& Kiecolt-Glaser, J. (1997). The importance of social versus temporal comparison appraisals among older adults. Journal of Applied Social Psychology, 27, 959-966.

Rosseel, Y. (2012). Lavaan: An R package for structural equation modeling. Journal of Statistical Software, 48, 1-36.

Scheinder, S. M., \& Schupp, J. (2014). Individual differences in social comparison and its consequences for life satisfaction: Introducing a short scale of the Iowa-Netherlands Comparison Orientation Measure. Social Indicators Research, 115, 767-789.

Smith, H. J., Pettigrew, T. F., Pippin, G. M., \& Bialosiewicz, S. (2012). Relative deprivation: A theoretical and meta-analytic review. Personality and Social Psychology Review, 16, 203-232.

Steenkamp, J.E.M., \& Baumgartner, H. (1998). Assessing measurement invariance in crossnational consumer research. Journal of Consumer Research, 25, 78-90.

Suls, J., \& Mullen, B. (1982). From the cradle to the grave: Comparison and self-evaluation across the life-span. In J. Suls (Ed.), Psychological perspectives on the self (Vol. 1, pp. 97-125). Hillsdale, NJ: Erlbaum. 
Table 1

Descriptive Statistics and Correlations among Measures.

\begin{tabular}{lccrrrr}
\hline Measures & $M(S D)$ & 1. & 2. & 3. & $3 \mathrm{a}$. & $3 \mathrm{~b}$. \\
\hline Study 1 & & & & & & \\
1. Age & $36.55(12.24)$ & -- & & & & \\
2. PRDS & $3.13(.95)$ & $-.20^{*}$ & $(.73)$ & & & \\
3. INCOM-11 & $3.34(.69)$ & $-.24^{*}$ & $.25^{*}$ & $(.85)$ & & \\
3a. Ability & $3.11(.86)$ & $-.29^{*}$ & $.33^{*}$ & $.92^{*}$ & $(.85)$ & \\
3b. Opinion & $3.58(.70)$ & $-.10^{*}$ & $.07^{*}$ & $.82^{*}$ & $.54^{*}$ & $(.72)$ \\
\hline
\end{tabular}

Study $2 \quad 18-30$ yrs. $60+$ yrs.

1. Age $\quad 25.21(3.19) \quad 64.98(3.75) \quad--$

2. PRDS $3.31(1.04) \quad 2.85(.99) \quad--\quad(.83)$

$\begin{array}{llllll}\text { 3. INCOM-6 } & 3.59(.62) & 3.03(.79) & -- & .20 * & (.82)\end{array}$

$\begin{array}{lllllll}\text { 3a. Ability } & 3.45(.84) & 2.73(.95) & -- & .27 * & .89 * & (.79)\end{array}$

3b. Opinion $3.73(.69) \quad 3.34(.88) \quad--\quad .06 \quad 3)^{*} \quad .48 * \quad(.82)$

Note. PRDS = Personal Relative Deprivation Scale, INCOM = Iowa Netherlands

Comparison Orientation Measure (11 and 6-item scales). Alpha reliabilities are presented in parentheses along the diagonals.

$* p<.05$. 
Table 2. Sample Characteristics by Age for Study 2

\begin{tabular}{lll}
\hline & Younger $(18-30)$ & Older $(60+)$ \\
\hline$M$ age $(S D) *$ & $25.21(3.19)$ & $64.98(3.75)$ \\
$\%$ Women & $35 \%$ & $38 \%$ \\
$\%$ White/Caucasian & $75.56 \%$ & $79.55 \%$ \\
$M$ income (SD) in $\$$ & $45.6 \mathrm{k}(37.07 \mathrm{k})$ & $53.35 \mathrm{k}(38.95 \mathrm{k})$ \\
$M$ Education* & $2.63(0.63)$ & $2.82(0.72)$ \\
\hline Note. $*=$ Difference between age groups, $p<.05$. &
\end{tabular}



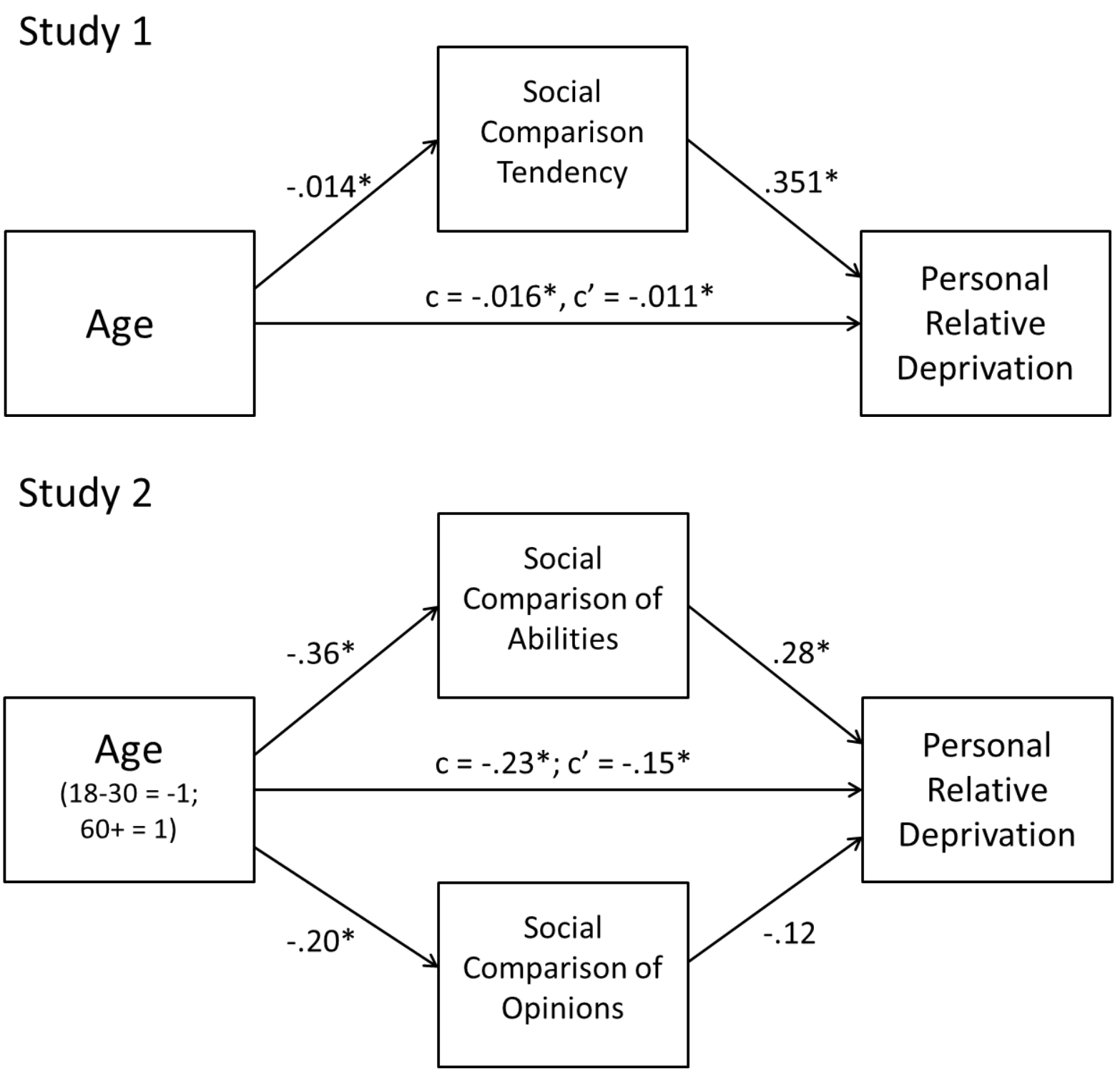

Figure 1. Mediational models of the relation between age and personal relative deprivation through social comparisons (Studies 1 and 2). Values depict unstandardized regression coefficients. ${ }^{*} p<.05$. 


\section{Supplementary Materials}

Here we report (1) the methods and results of an additional study exploring age-related differences in social comparison tendencies and personal relative deprivation (PRD) and (2) provide more information about the multi-group CFAs mentioned in the main text (see Table S1).

\section{Study S1. Exploratory study of age-related differences in social comparison tendencies and personal relative deprivation.}

\section{Participants}

Method

Participants were 480 adults ( $57 \%$ female) in the United Kingdom who were recruited through an Internet market research panel (PureProfile.com). Twenty additional participants were removed from the final dataset because they completed the survey twice or were not in the UK (the latter participants were removed because we asked participants to report their annual household income in pound sterling; see below). Our initial strategy was to recruit 250 participants within each of the age categories of 18-24 and 60+ years old, but we extended eligibility to 18 to 30 year olds for the younger category because there were a limited number of participants available in the online panel within the 18-24 range.

\section{Procedure and measures}

Participants first reported their age to determine their eligibility to participate. Eligible participants completed the 11-item INCOM and the PRDS (per Study 1 in the main text). Participants then reported their gender and annual household income with an 18-point ordinal scale including values ranging from 1 (less than $£ 5,000$ ) to 18 ( $£ 85,001$ and above), with each option spanning $£ 4,999$. Income responses were coded using the category mid-points, with the value for the category at the top of the scale being the median-based estimator described by Parker and Fenwick (1983). Annual income was included as a control variable to test whether age-related differences might be due to differences in wealth between the age groups.

\section{Results}

\section{Confirmatory Factor Analyses and Tests of Measurement Invariance}

Following Gibbons and Buunk (1999), our a priori analysis strategy was to examine age-related differences in social comparison tendency using the full INCOM (i.e., assuming a single factor model). However, consistent with Schneider and Schupp's (2014) findings, CFAs showed that the one-factor solution for the INCOM provided worse fit $\chi^{2}=377.27, \mathrm{df}=43, p<.001 ; \mathrm{CFI}=.877 ; \mathrm{TLI}=.843$; RMSEA $=0.127 ;$ SRMR $=.065$ ) than the two-factor solution (i.e., with the ability and opinion subscales as distinct but correlated factors; $\chi^{2}=125.64, \mathrm{df}=42, p<.001 ; \mathrm{CFI}=.969 ; \mathrm{TLI}=.960$; RMSEA $=.064$; SRMR $=.039) .{ }^{2}$ For these analyses, correlations between the error variances for the two reversed worded items were specified (cf. Schneider \& Schupp, 2014; see Brown \& Moore, 2012). Further, the items from Schneider and Schupp's revised 6-item version of the INCOM (INCOM-6; which includes three items from each of the original ability and opinion subscales) showed excellent fit $\left(\chi^{2}=18.89, \mathrm{df}=15, p=.015 ; \mathrm{CFI}=.992 ; \mathrm{TLI}=.985 ; \mathrm{RMSEA}=.053 ;\right.$ SRMR $=.03$ ).

\footnotetext{
2 The same pattern was observed in analyses of the data from Study 1 of the main text: the one-factor solution for the full INCOM showed worse fit $\left(\chi^{2}=1124.57, \mathrm{df}=43, p<.001 ; \mathrm{CFI}=.800 ; \mathrm{TLI}=.745 ; \mathrm{RMSEA}=0.14 ; \mathrm{SRMR}\right.$ $=.14)$ than the two-factor solution $\left(\chi^{2}=271.08, \mathrm{df}=42, p<.001 ; \mathrm{CFI}=.958 ; \mathrm{TLI}=.945 ; \mathrm{RMSEA}=0.065 ; \mathrm{SRMR}=\right.$ .049).
} 
Below we report age-related differences using the full INCOM (per our initial analysis plan) along with exploratory analyses for the ability and opinion subscales of the INCOM-6.

Shown in Table S1, a model comparison approach using Multi-group Confirmatory Factor Analyses (MCFA) for testing measurement invariance showed that both the INCOM-6 and the PRDS were age invariant in terms of equivalence of the factor loadings (configural vs. metric model). Tests of the equivalence of factor loadings and intercepts (scalar vs. metric invariance) across age groups showed that although the $\Delta \chi^{2}$ were statistically significant for both the PRDS and INCOM-6, changes in CFI between invariance models were less than .01, implying that the scalar invariance assumption still holds (Cheung \& Rensvold, 2002). Nonetheless, at least partial scalar invariance was established in terms of both $\Delta \chi^{2}$ and $\Delta C F I$ when we relaxed the equality of intercepts constraint for one item for each of the measures (see Table S1). Invariance testing for the full 11-item INCOM revealed that the configural invariance model fit the data poorly $\left(\chi^{2}=406.17, \mathrm{df}=86, p<.001 ; \mathrm{CFI}=.856\right.$; RMSEA $=$ $0.125)$; therefore, no further invariance model comparisons were performed.

Table S1. Hierarchical Multi-group CFA models testing measurement invariance for age for Study 2 (main text) and Study S1.

\begin{tabular}{|c|c|c|c|c|c|c|}
\hline Model & $\chi^{2}$ & $\mathrm{df}$ & $\Delta \chi^{2}$ & CFI & $\Delta \mathrm{CFI}$ & RMSEA \\
\hline \multicolumn{7}{|c|}{ Main Text Study 2, PRDS } \\
\hline Configural & 15.51 & 8 & & .991 & & .073 \\
\hline Metric & 18.81 & 12 & 3.31 & .992 & -.001 & .056 \\
\hline $\begin{array}{l}\text { Scalar } \\
\text { Main Text Study } 2\end{array}$ & $\begin{array}{l}22.88 \\
\text { INCOM-6 }\end{array}$ & 16 & 4.07 & .992 & .000 & .049 \\
\hline Configural & $28.29 *$ & 16 & & .983 & & .066 \\
\hline Metric & $32.28^{*}$ & 20 & 3.99 & .983 & .000 & .059 \\
\hline Scalar & $50.69 *$ & 24 & $18.41^{*}$ & .963 & .020 & .079 \\
\hline $\begin{array}{l}\text { Partial scalar } \\
\text { (except INCOM3) }\end{array}$ & $32.59 *$ & 23 & .31 & .987 & -.004 & .048 \\
\hline
\end{tabular}

\section{Study S1, PRDS}

$\begin{array}{lllllll}\text { Configural } & 36.61^{*} & 8 & & .972 & & .122 \\ \text { Metric } & 45.26^{*} & 12 & 8.65 & .968 & .005 & .107 \\ \text { Scalar } & 56.23^{*} & 16 & 10.98^{*} & .961 & .007 & .102 \\ \text { Partial scalar } & 49.64^{*} & 15 & 4.38 & .966 & .001 & .098 \\ \text { (except PRD3) } & & & & & & \end{array}$

\section{Study S1, INCOM-6}

$\begin{array}{lllllll}\text { Configural } & 37.04^{*} & 16 & & .980 & & .074 \\ \text { Metric } & 46.53^{*} & 20 & 9.49 & .975 & .005 & .074 \\ \text { Scalar } & 59.18^{*} & 24 & 12.65^{*} & .967 & .008 & .078 \\ \text { Partial scalar } & & & & & & \\ \text { (except INCOM2) } & 49.01^{*} & 23 & 2.48 & .976 & .000 & .069\end{array}$

Note. PRDS = Personal Relative Deprivation Scale. INCOM-6 $=6$-item version of the lowaNetherlands Comparison Orientation Scale. INCOM3 = "I am not the type of person who compares 
often with others". PRD3 = "I feel resentful when I see how prosperous other people like me seem to be". INCOM2 = "I always pay a lot of attention to how I do things compared with how others do things". * $p<.05$.

\section{Age Differences in INCOM and PRD}

Shown in Table S2, the older (vs. younger) adults demonstrated weaker tendency to socially compare for the full 11-item INCOM, $t(478)=12.23, p<.001, d=1.12$, the INCOM- 6 ability subscale, $t(478)=13.23, p<.001, d=1.21$, and the INCOM-6 opinion subscale, $t(478)=7.38, p<.001, d=.68$. The older participants also reported lower PRD, $t(478)=10.52, p<.001, d=.96$. Analyses controlling for annual income and gender produced virtually identical results.

Comparisons of the latent means revealed the same pattern of age-related differences for PRD $(p<$ $.001, d=1.14$; allowing the non-invariant intercept for the PRD3 item to be different, $d=1.07$ ), social comparison of abilities ( $p<.001, d=1.39$; allowing the non-invariant intercept for the INCOM2 item to be different, $d=1.55)$, and social comparisons of opinions $(p<.001, d=.77)$.

Table S2. Descriptive Statistics and Correlations among Measures.

\begin{tabular}{|c|c|c|c|c|c|c|c|}
\hline \multirow[t]{2}{*}{ Measures } & \multicolumn{2}{|c|}{$M(S D)$} & \multirow[t]{2}{*}{1.} & \multirow[t]{2}{*}{2.} & \multirow[t]{2}{*}{3.} & \multirow[t]{2}{*}{4.} & \multirow[t]{2}{*}{5.} \\
\hline & $\begin{array}{l}18-30 \text { yrs. ( } n \\
=234)\end{array}$ & $\begin{array}{l}60+y r s . \\
(n=246)\end{array}$ & & & & & \\
\hline 1. Age & $25.31(2.80)$ & $66.63(5.22)$ & -- & & & & \\
\hline 2. PRDS & $3.29(.85)$ & $2.50(.79)$ & -- & $(.74)$ & & & \\
\hline 3. INCOM-11 & $3.47(.61)$ & $2.71(.74)$ & -- & $.37^{*}$ & $(.90)$ & & \\
\hline $\begin{array}{r}\text { 4. INCOM-6 } \\
\text { (Ability) }\end{array}$ & $3.37(.86)$ & $2.33(.87)$ & -- & $.40^{*}$ & $.88 *$ & $(.82)$ & \\
\hline $\begin{array}{l}\text { 5. INCOM-6 } \\
\text { (Opinion) }\end{array}$ & $3.60(.72)$ & $3.04(.92)$ & -- & $.22^{*}$ & $.83^{*}$ & $.58^{*}$ & $(.83)$ \\
\hline
\end{tabular}

Note. PRDS= Personal Relative Deprivation Scale, INCOM = lowa Netherlands

Comparison Orientation Measure (11 and 6-item scales). Alpha reliabilities are presented in parentheses along the diagonals. ${ }^{*} p<.05$.

\section{Mediation analyses}

Bootstrapped mediation analyses showed that the full INCOM mediated the effect of age on PRD $(10,000$ resamples; indirect effect $=-.09,95 \% \mathrm{BCa} \mathrm{Cl}$ of -.139 and -.047$)$. Consistent with the pattern reported in Study 2 of the main text, multiple mediation analyses showed that social comparison of abilities (indirect effect $=-.117,95 \% \mathrm{BCa} \mathrm{Cl:}-.174,-.069$ ), but not social comparison of opinions (indirect effect $=.006,95 \% \mathrm{BCa} \mathrm{Cl}:-.022, .033$ ), mediated the effect of age on PRD (see Figure S1). 


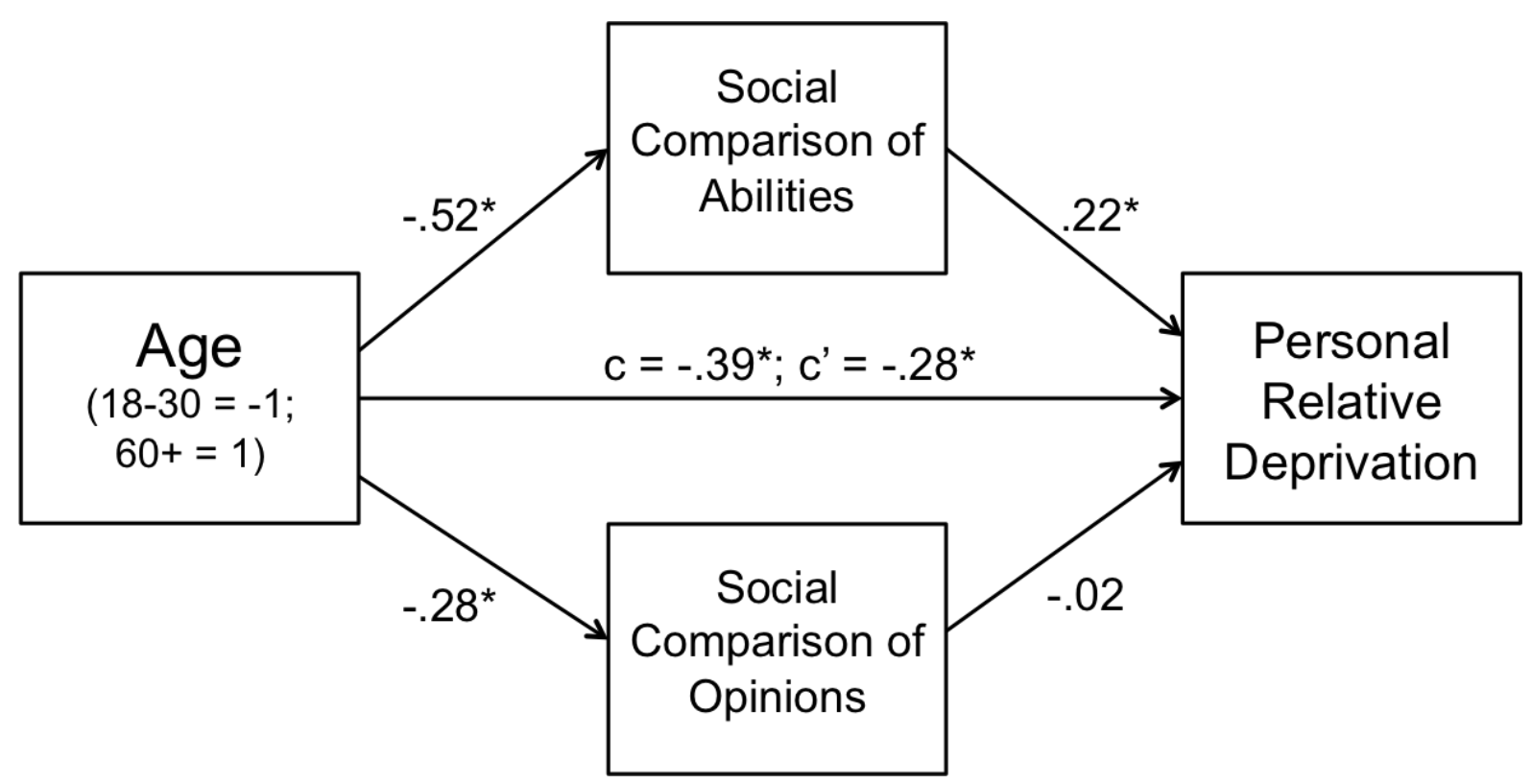

Figure S1. Mediational model of the relation between age and personal relative deprivation through social comparisons of abilities and opinions (Study S1). Values depict unstandardized regression coefficients. ${ }^{*} p<.05$.

\section{References}

Brown, T. A. \& Moore, M. T. (2012). Confirmatory factor analysis. In R. Hoyle (Eds.). Handbook of Structural Equation Modeling. (pp. 361-379). New York, NY: The Guilford Press.

Cheung, G. W., \& Rensvold, R. B. (2002). Evaluating goodness-of-fit indexes for testing measurement invariance. Structural Equation Modeling, 9, 233-255

Gibbons, F. X., \& Buunk, B. P. (1999). Individual differences in social comparison: Development of a scale of social comparison orientation. Journal of Personality and Social Psychology, 76, 129142.

Parker, R. N., \& Fenwick, R. (1983). The Pareto curve and its utility for open-ended income distributions in survey research. Social Forces, 61, 872-885.

Scheinder, S. M., \& Schupp, J. (2014). Individual differences in social comparison and its consequences for life satisfaction: Introducing a short scale of the lowa-Netherlands Comparison Orientation Measure. Social Indicators Research, 115, 767-789. 